\title{
Graphs with chromatic numbers strictly less than their colouring numbers*
}

\author{
Xuding Zhu \\ Department of Mathematics, Zhejiang Normal University, Zhejiang, P. R. China, and \\ Department of Applied Mathematics, National Sun Yat-sen University, Taiwan
}

Received 26 April 2010, accepted 26 August 2010, published online 18 October 2010

\begin{abstract}
The colouring number of a graph $G$, defined as $\operatorname{col}(G)=1+\max _{H \subseteq G} \delta(H)$, is an upper bound for its chromatic number. In this note, we prove that it is NP-complete to determine whether an arbitrary graph $G$ has chromatic number strictly less than its colouring number.
\end{abstract}

Keywords: Chromatic number, colouring number, Szekeres-Wilf inequality, NP-completeness.

Math. Subj. Class.: 05C15

\section{Main result}

An easy upper bound for the chromatic number of a graph $G$ is that $\chi(G) \leq \Delta(G)+1$, where $\Delta(G)$ is the maximum degree of $G$. This upper bound is sharp; however, Brooks' Theorem [1] shows that the bound is only attained by complete graphs and odd cycles. The colouring number $\operatorname{col}(G)$ of $G$ is defined as $\operatorname{col}(G)=1+\max _{H \subseteq G} \delta(H)$, where $\delta(H)$ is the minimum degree of $H$. The Szekeres-Wilf inequality $\chi(G) \leq \operatorname{col}(G)$ gives a better upper bound for $\chi(G)$ [3]. This upper bound is also an easy bound, as the colouring number of $G$ can be calculated in linear time as follows: Assume $G$ has $n$ vertices. Let $G_{0}=G$, and for $1 \leq i \leq n-1$, let $G_{i}=G_{i-1}-v_{i}$, where $v_{i}$ is a vertex of minimum degree in $G_{i-1}$. Then $\operatorname{col}(G)=\max \delta\left(G_{i}\right)+1$. One naturally wonders if there is an analog of Brooks' Theorem that gives a simple characterization of all the graphs $G$ for which the Szekeres-Wilf inequality holds with equality. This note shows that it is unlikely to have a simple characterization for such graphs, as it is NP-complete to decide whether $\chi(G)<\operatorname{col}(G)$ for an arbitrary graph $G$.

\footnotetext{
* In memory of Michael O. Albertson.

E-mail address: zhu@math.nsysu.edu.tw (Xuding Zhu)
} 
Theorem 1.1. The following decision problem is NP-complete:

Instance: A graph $G$.

Question: Is $\chi(G)<\operatorname{col}(G)$ ?

Proof. As $\operatorname{col}(G)$ can be computed in linear time, it is obvious that the problem is in NP. In the following, we reduce the well-known NP-complete 3 -colourability problem to the above decision problem.

Suppose we need to decide whether a given graph $G$ is 3 -colourable. If $\operatorname{col}(G) \leq 3$, then $\chi(G) \leq \operatorname{col}(G) \leq 3$ and $G$ is 3-colourable.

Assume $\operatorname{col}(G)=k \geq 4$. We construct a new graph $G^{\prime}$ as follows: Take a copy of $G$. For each 4-subset $X$ of $V(G)$, add a set $U_{X}$ of $k-4$ new vertices. Add edges to connect every pair of vertices in $U_{X}$ (so that $U_{X}$ induces a copy of $K_{k-4}$ ), and connect each vertex of $U_{X}$ to every vertex of $X$ (the vertices in $X$ are 'old' vertices in $V(G)$ ). For different 4-subsets $X, X^{\prime}$ of $V(G), U_{X}$ and $U_{X^{\prime}}$ are disjoint. Also $U_{X}$ is disjoint from $V(G)$. So if $G$ has $n$ vertices, then $G^{\prime}$ has $n+\left(\begin{array}{l}n \\ 4\end{array}\right) \times(k-4) \leq n^{5}$ vertices. Note that if $k=4$, then $G^{\prime}=G$.

We shall show that $G$ is 3-colourable if and only if $\chi\left(G^{\prime}\right)<\operatorname{col}\left(G^{\prime}\right)$. Since all the new vertices (i.e., vertices not in $V(G)$ ) have degree $k-1$, we know that $\operatorname{col}\left(G^{\prime}\right)=\operatorname{col}(G)=k$. If $k=4$, then $G=G^{\prime}$ and $\chi\left(G^{\prime}\right)<\operatorname{col}\left(G^{\prime}\right)=4$ is equivalent to $G=G^{\prime}$ is 3-colourable. Assume $k \geq 5$. If $G$ has a 3 -colouring $f$, then we can extend $f$ to a $(k-1)$-colouring of $G^{\prime}$. This is so, because if $v$ is an added vertex, then $v \in U_{X}$ for some 4-subset $X$ of $V(G)$. The vertex $v$ has $k-1$ neighbours, and at least two of the neighbours of $v$ in $X$ are coloured by the same colour. So we can choose a colour for $v$ which is not used by any of its neighbours.

Conversely, assuming $G$ is not 3 -colourable, we shall show that $G^{\prime}$ is not $(k-1)$ colourable. Assume to the contrary that $f$ is a $(k-1)$-colouring of $G^{\prime}$. Since $G$ is not 3 -colourable, the restriction of $f$ to $V(G)$ uses at least 4 colours. So there is a 4 -subset $X$ of $V(G)$ such that $|f(X)|=4$. As each vertex of $X$ is adjacent to all the vertices in $U_{X}$, none of the 4 colours in $f(X)$ can be used by any vertex in $U_{X}$. So the number of colours that can be used on the vertices of $U_{X}$ is $\left|U_{X}\right|-1$. This is impossible, as $U_{X}$ induces a complete graph.

So the problem of deciding whether $G$ is 3-colourable is reduced to the problem of deciding whether $\chi\left(G^{\prime}\right)=\operatorname{col}\left(G^{\prime}\right)$. As $\left|V\left(G^{\prime}\right)\right| \leq|V(G)|^{5}$, the reduction is polynomial. So it is NP-complete to decide whether an arbitrary graph $G^{\prime}$ satisfies the strict inequality $\chi\left(G^{\prime}\right)<\operatorname{col}\left(G^{\prime}\right)$.

\section{Acknowledgement}

The author thanks Professor Wilf for asking this question during the coffee break at the conference "CoNE Revisited: Celebrating the Inspirations of Michael O. Albertson".

\section{References}

[1] R. L. Brooks, On colouring the nodes of a network, Proc. Cambridge Philos. Soc. 37 (1947), 194-197.

[2] M. R. Garey and D. S. Johnson, Computers and Intractability: A Guide to the Theory of NPCompleteness, W. H. Freeman, New York, 1979. 
[3] G. Szekeres and H. S. Wilf, An inequality for the chromatic number of a graph, J. Comb. Theory 4 (1968), 1-3. 